\title{
Highly Stereoselective One-Pot Procedure to Prepare Unsymmetrical Bis- and Tris-chalcogenide Alkenes via Addition of Chalcogens to Alkynes
}

Caroline C. Schneider, Benhur Godoy, Marina Prigol, Cristina W. Nogueira, Gilson Zeni*

${ }^{a}$ Laboratório de Síntese, Reatividade, Avaliação, Toxicológica e Farmacológica de Organocalcogenios-CCNE-UFSM-97105-900-Santa Maria-RS-Brazil

gzeni@quimica.ufsm.br

\section{SUPPORTING INFORMATION}

CONTENTS

Materials and Methods

Selected Spectra

S3 


\section{Materials and Methods}

Proton nuclear magnetic resonance spectra $\left({ }^{1} \mathrm{H}\right.$ NMR) were obtained at 400 MHz. Spectra were recorded in $\mathrm{CDCl}_{3}$ solutions. Chemical shifts are reported in ppm, referenced to the solvent peak of $\mathrm{CDCl}_{3}$ or tetramethylsilane (TMS) as the external reference. Data are reported as follows: chemical shift $(\delta)$, multiplicity, coupling constant $(J)$ in Hertz and integrated intensity. Carbon-13 nuclear magnetic resonance spectra $\left({ }^{13} \mathrm{C}\right.$ NMR) were obtained at $100 \mathrm{MHz}$. Spectra were recorded in $\mathrm{CDCl}_{3}$ solutions. Chemical shifts are reported in ppm, referenced to the solvent peak of $\mathrm{CDCl}_{3}$. Abbreviations to denote the multiplicity of a particular signal are s (singlet), d (doublet), $\mathrm{t}$ (triplet), q (quartet), quint (quintet), sex (sextet) and m (multiplet). High-resolution mass spectra were recorded on a double focusing magnetic sector mass spectrometer using EI at $70 \mathrm{eV}$. Mass spectra were recorded on an electron impact and chemical ionization positive ion mode. Reagents and solvents were handled using standard syringe techniques. Temperatures above room temperature were maintained by use of a mineral oil bath with an electrically heated coil connected to a controller.

Column chromatography was performed using silica gel (230-400 mesh) following the methods described by Still. ${ }^{1}$ Thin layer chromatography (TLC) was performed using silica gel $\mathrm{GF}_{254}, 0.25 \mathrm{~mm}$ thickness. For visualization, TLC plates were either placed under ultraviolet light, or stained with iodine vapor, or acidic vanillin.

\footnotetext{
${ }^{1}$ Still, W. C.; Kahan, M.; Mitra, A. J. Org. Chem. 1978, 43, 2923-2925.
} 


\section{$\underline{\text { Selected Spectra }}$}

PDF created with pdfFactory trial version www.pdffactory.com 


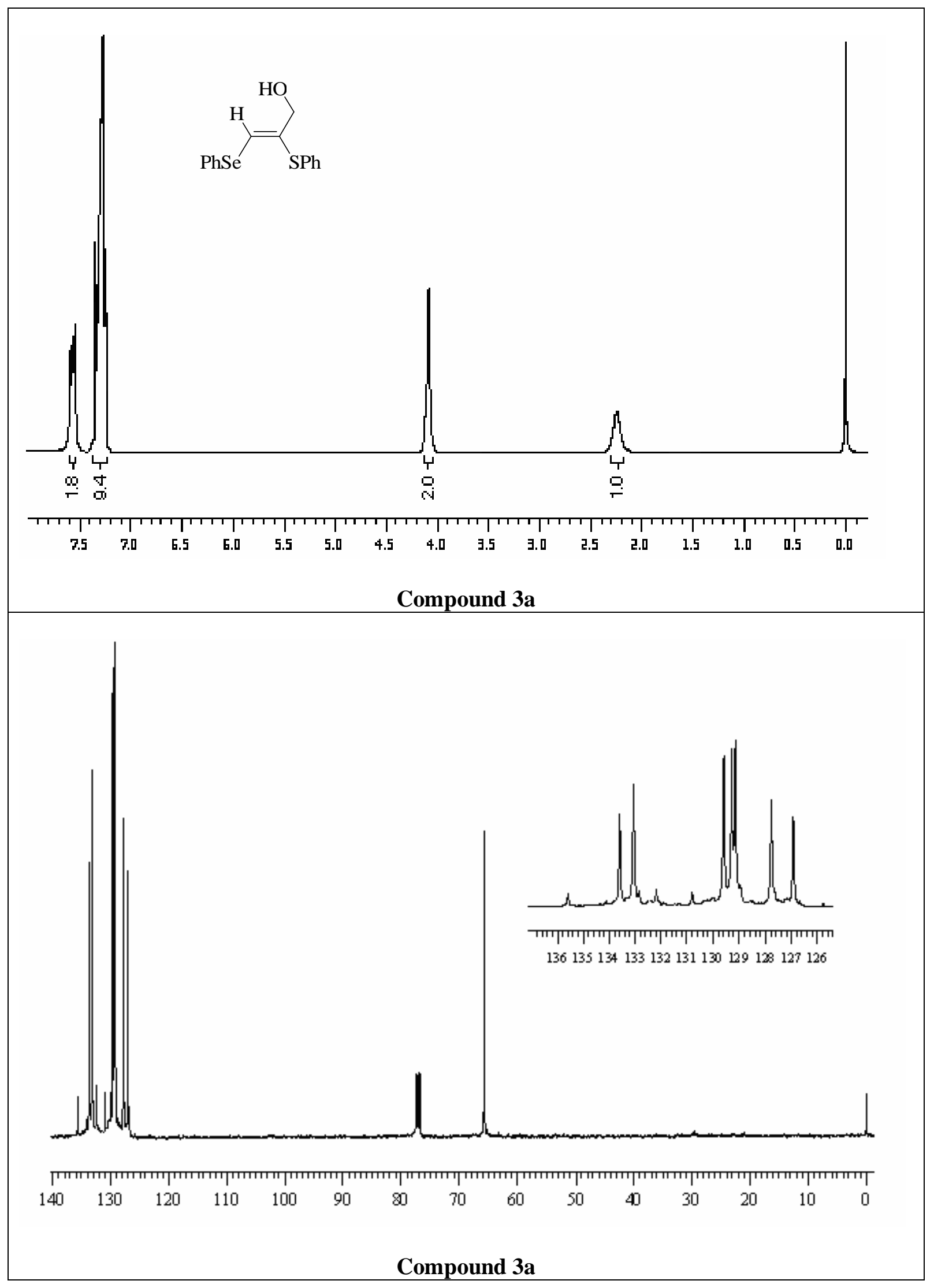




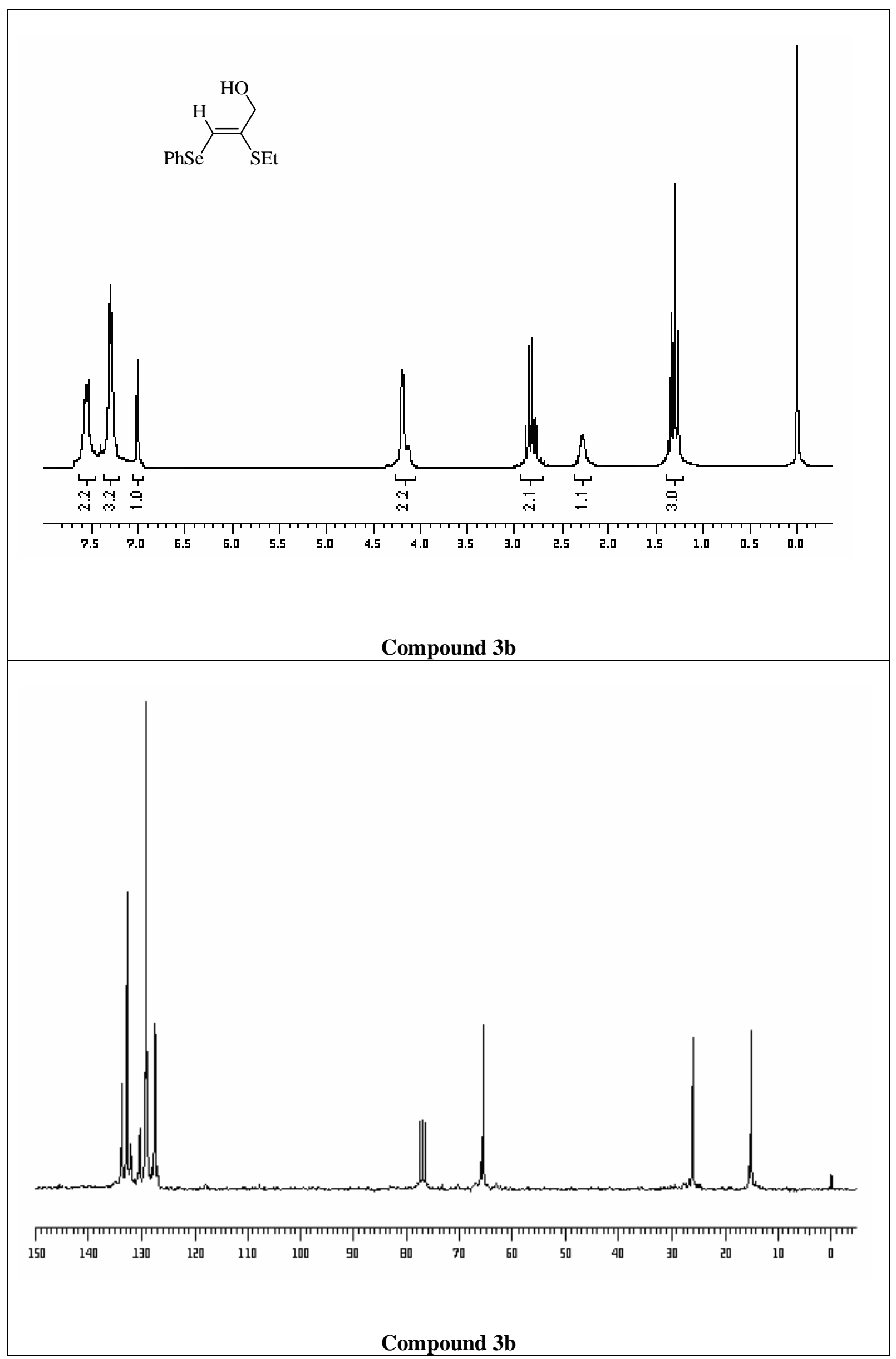




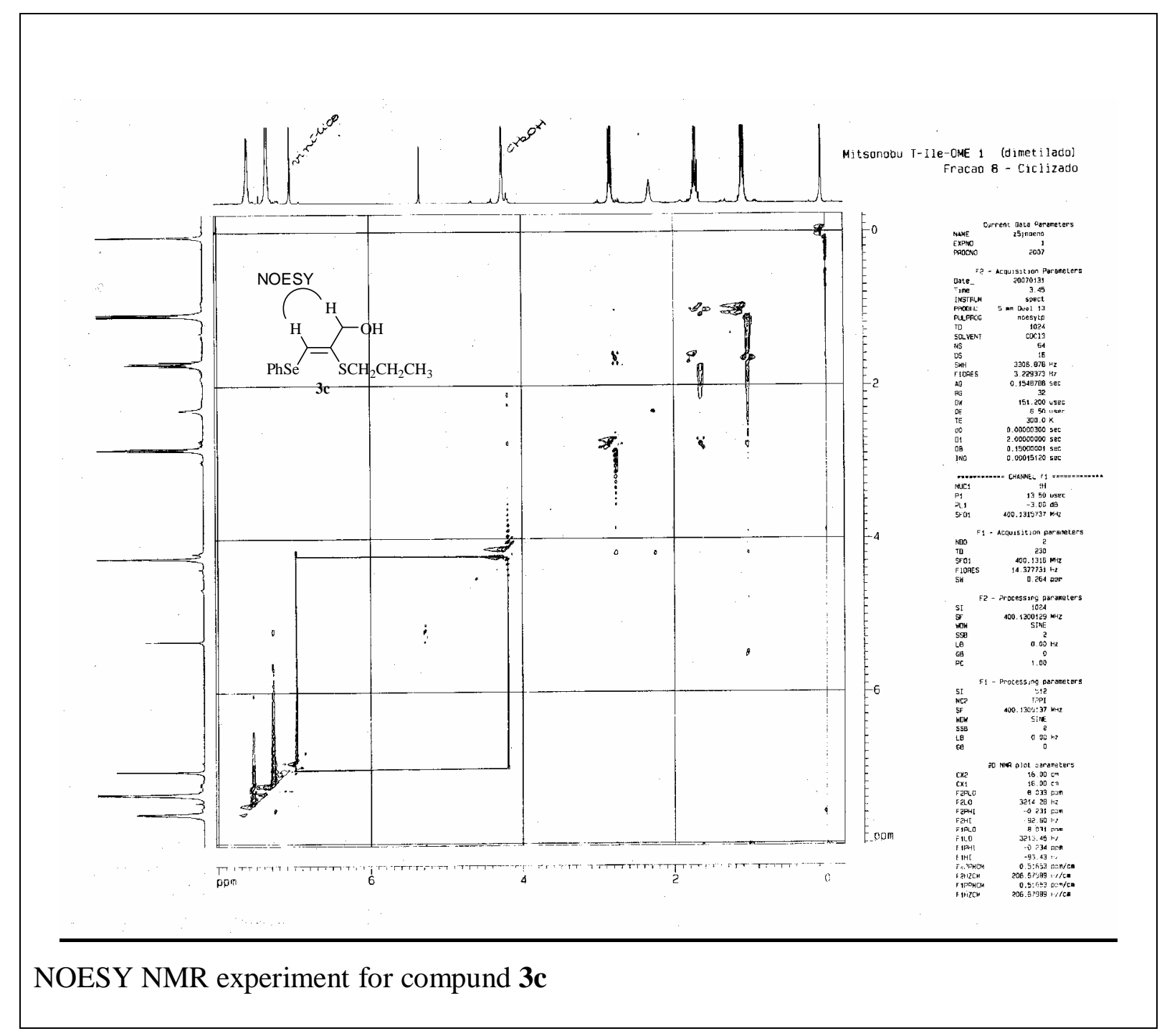




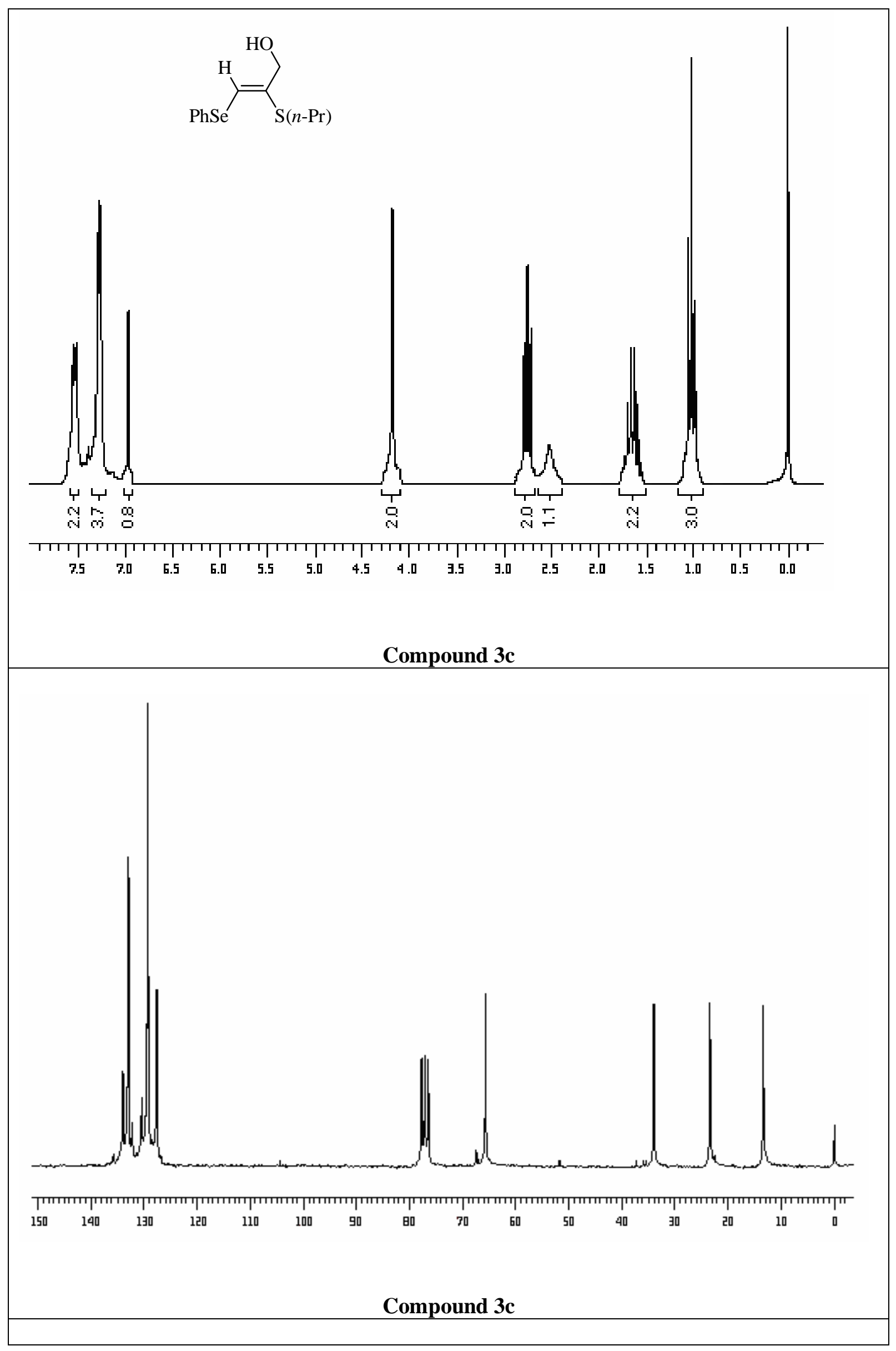




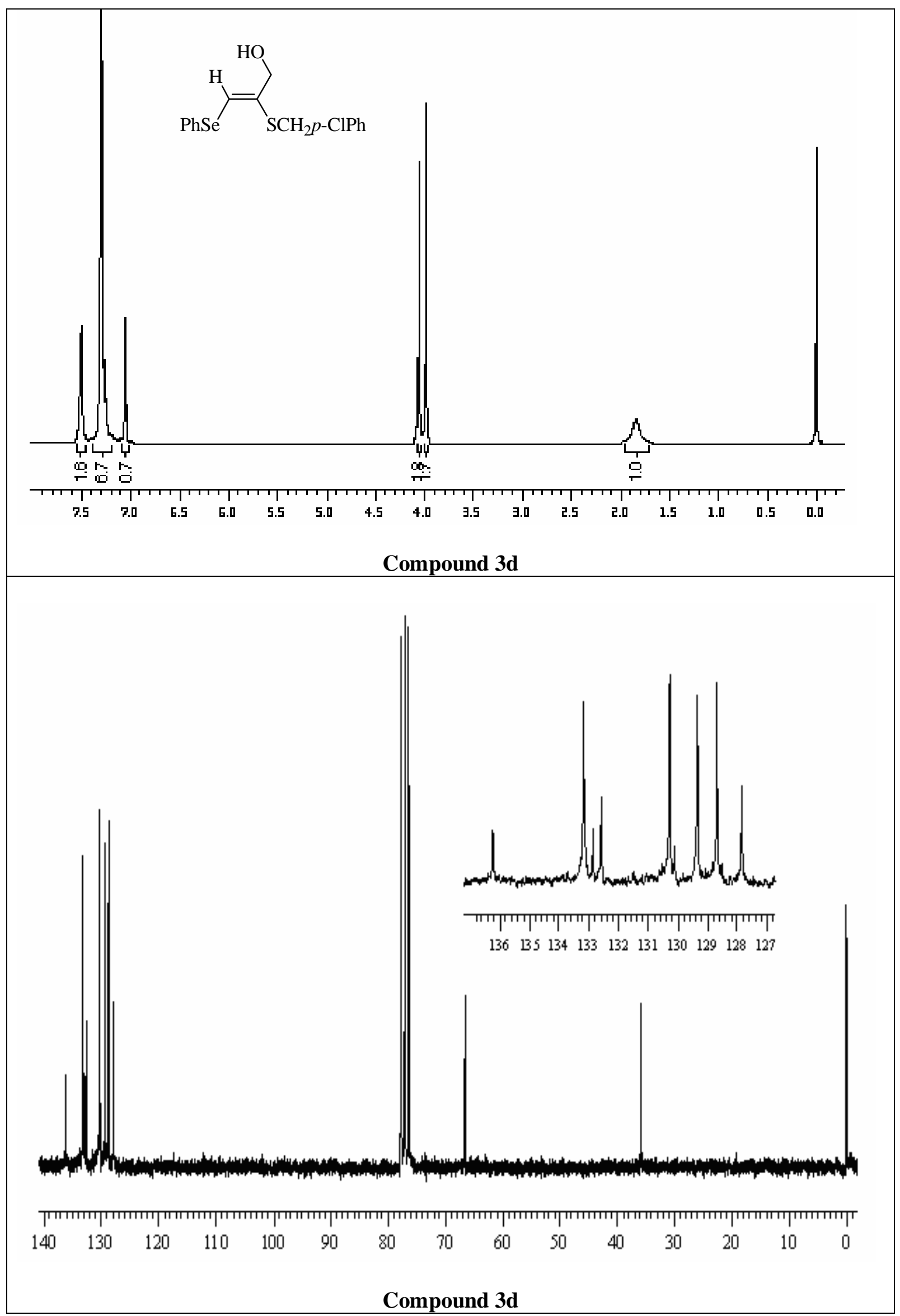

Compound 3d 


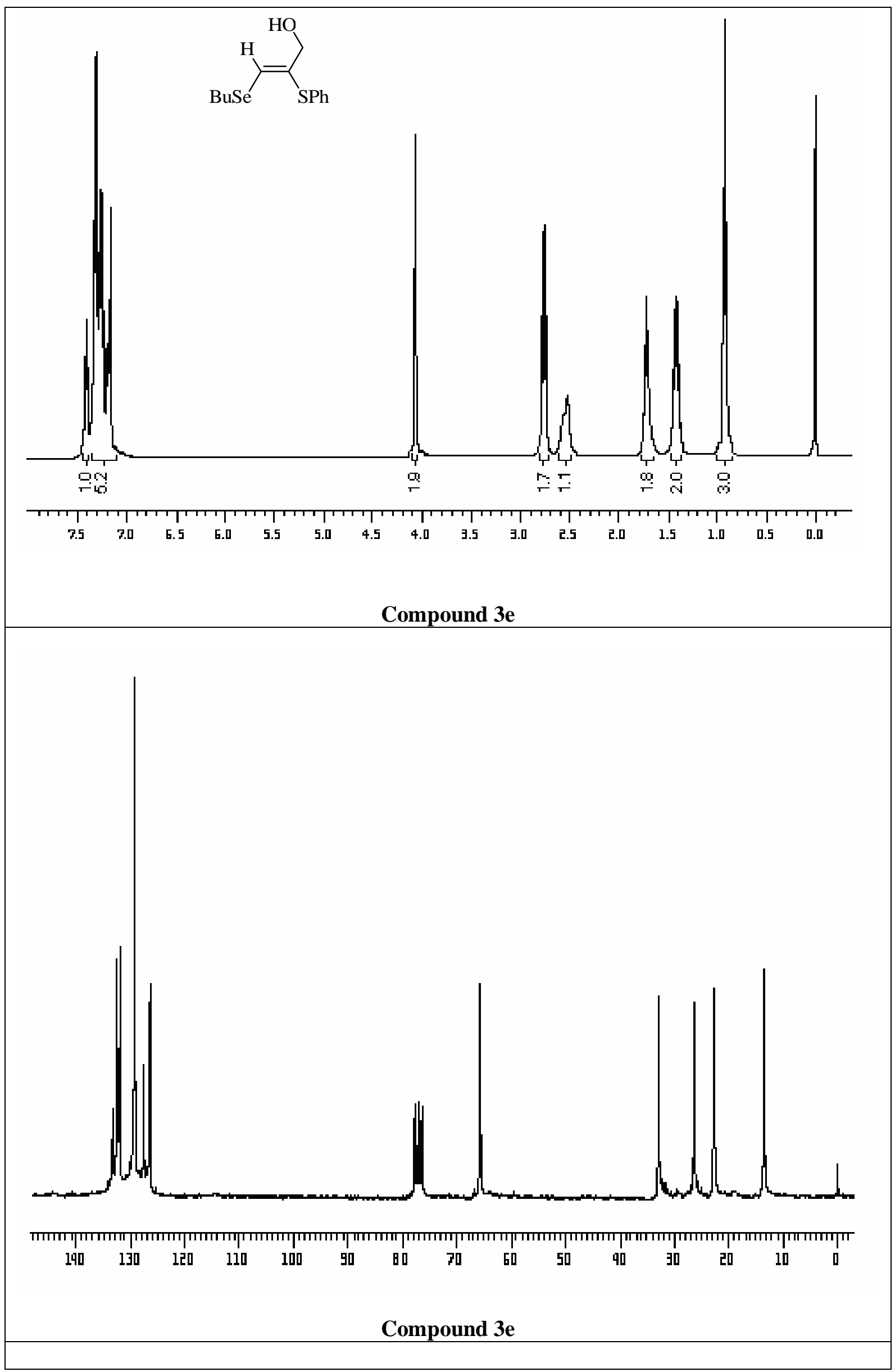




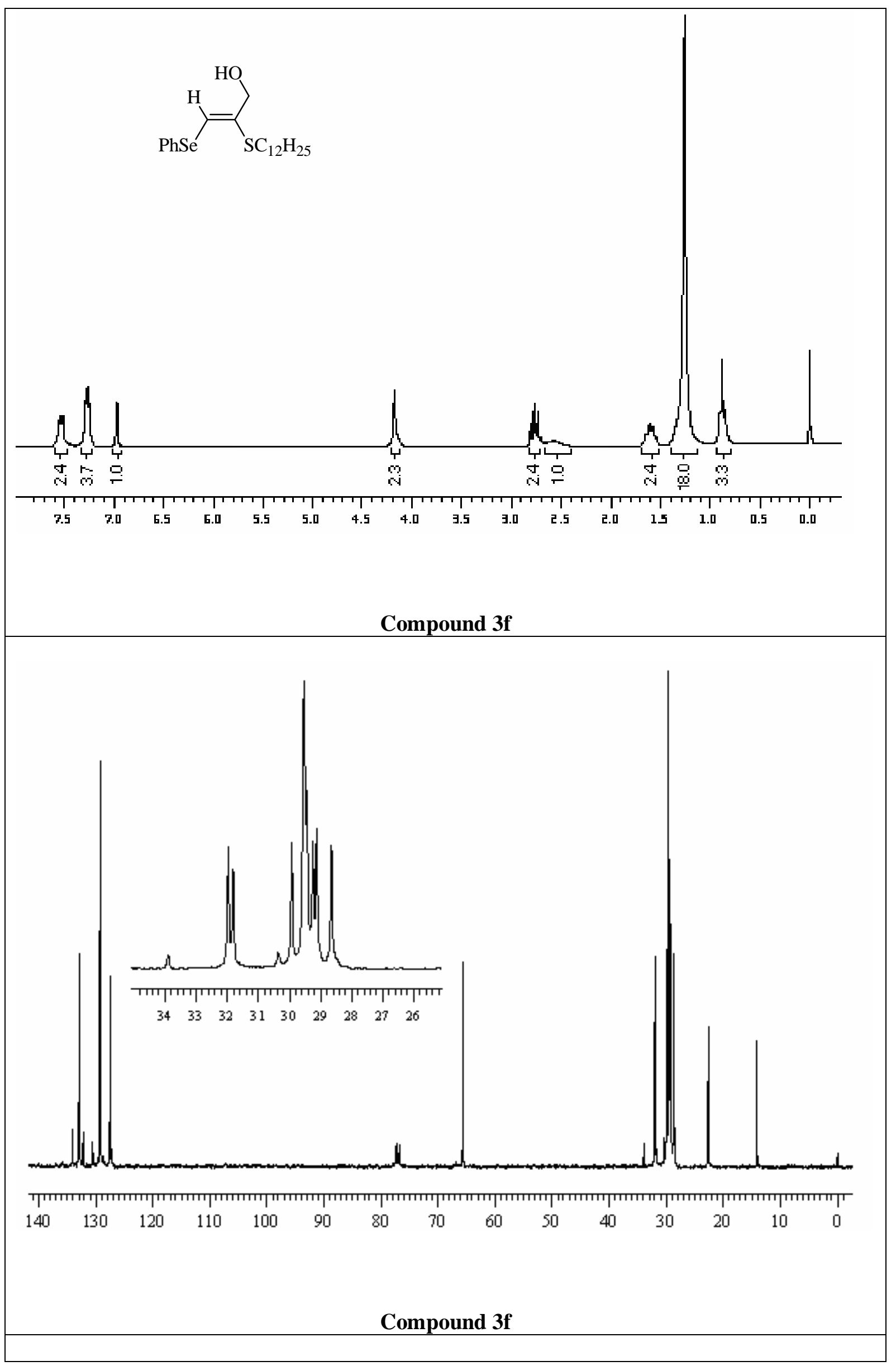




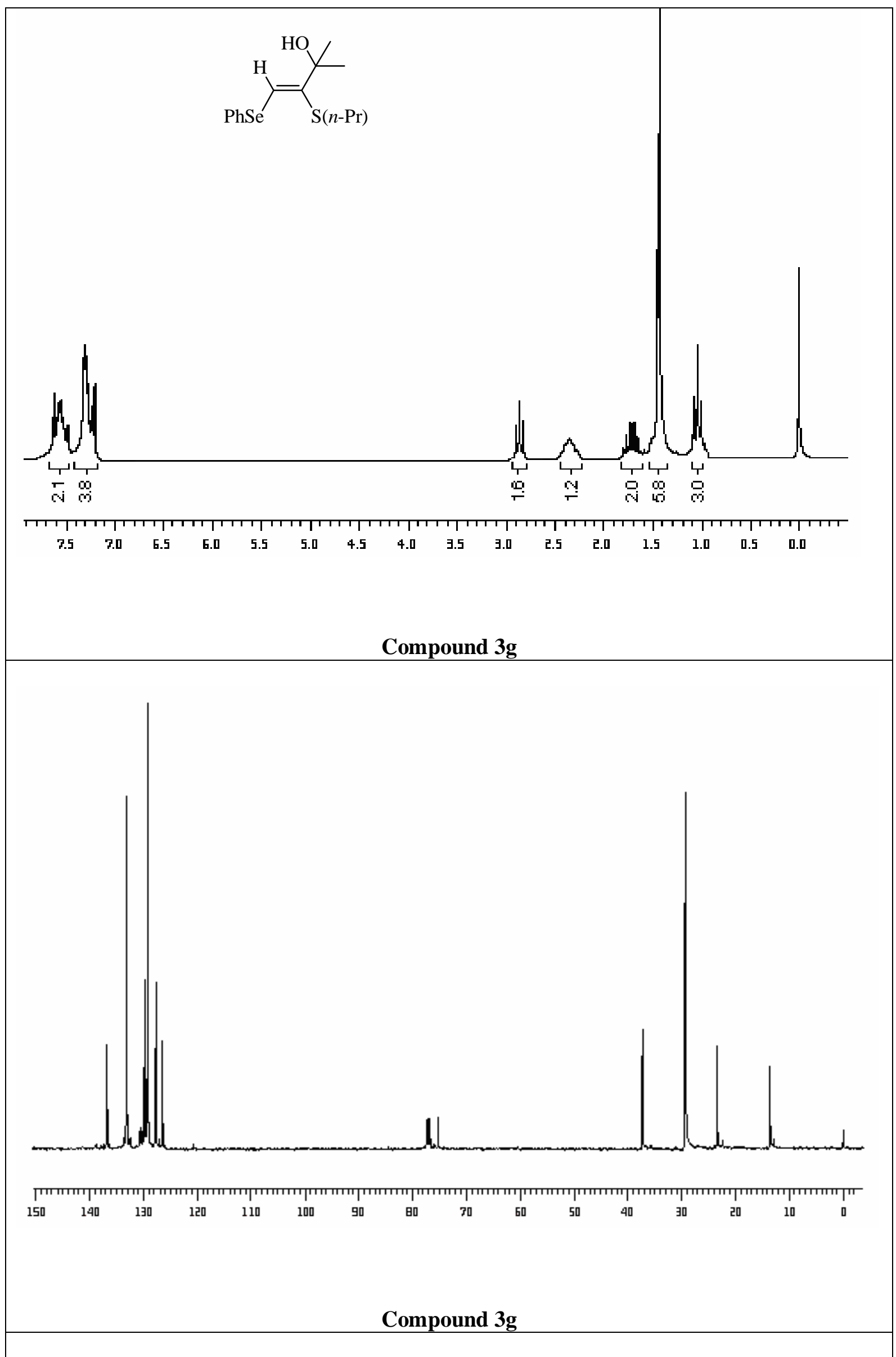




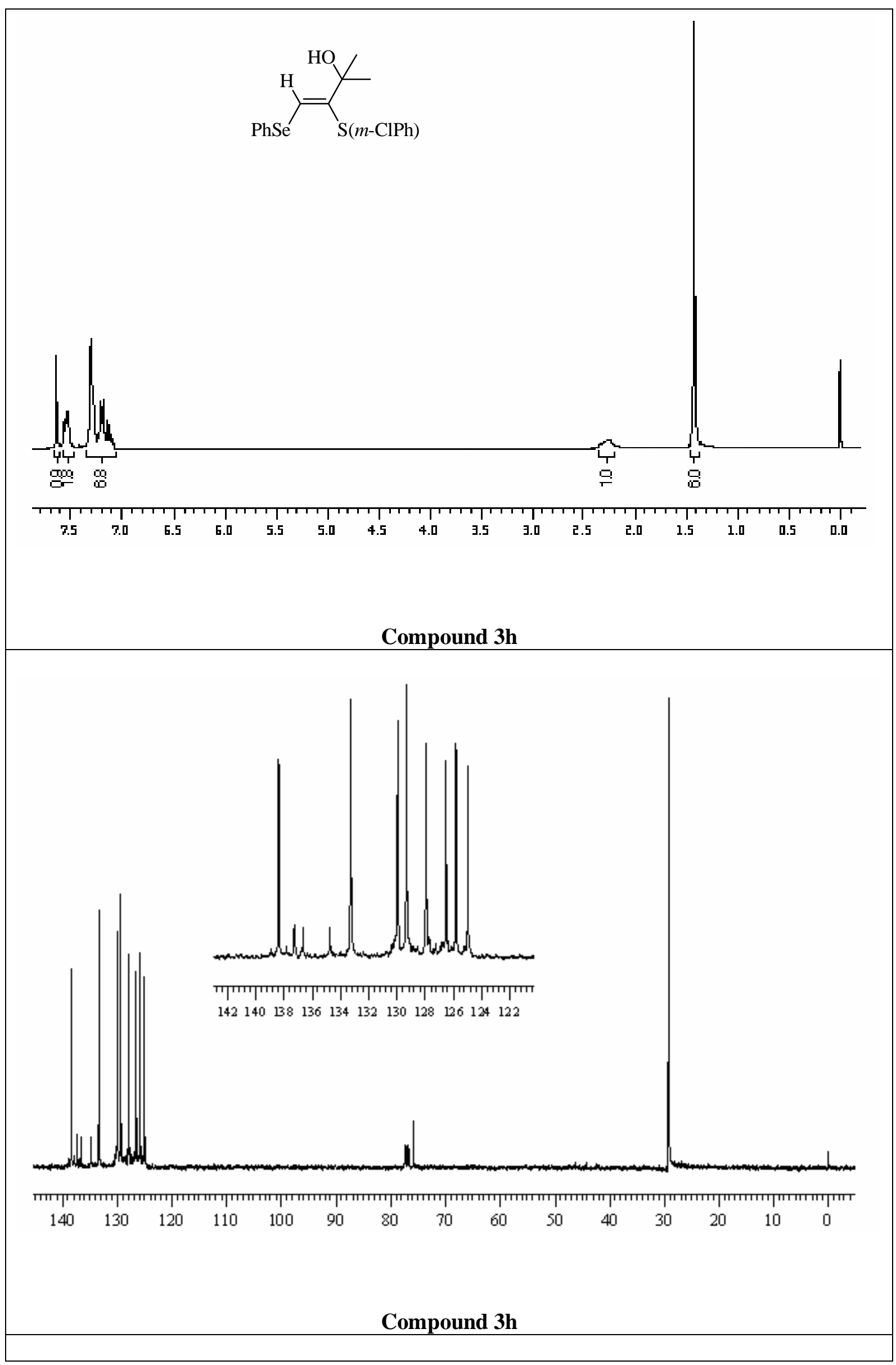




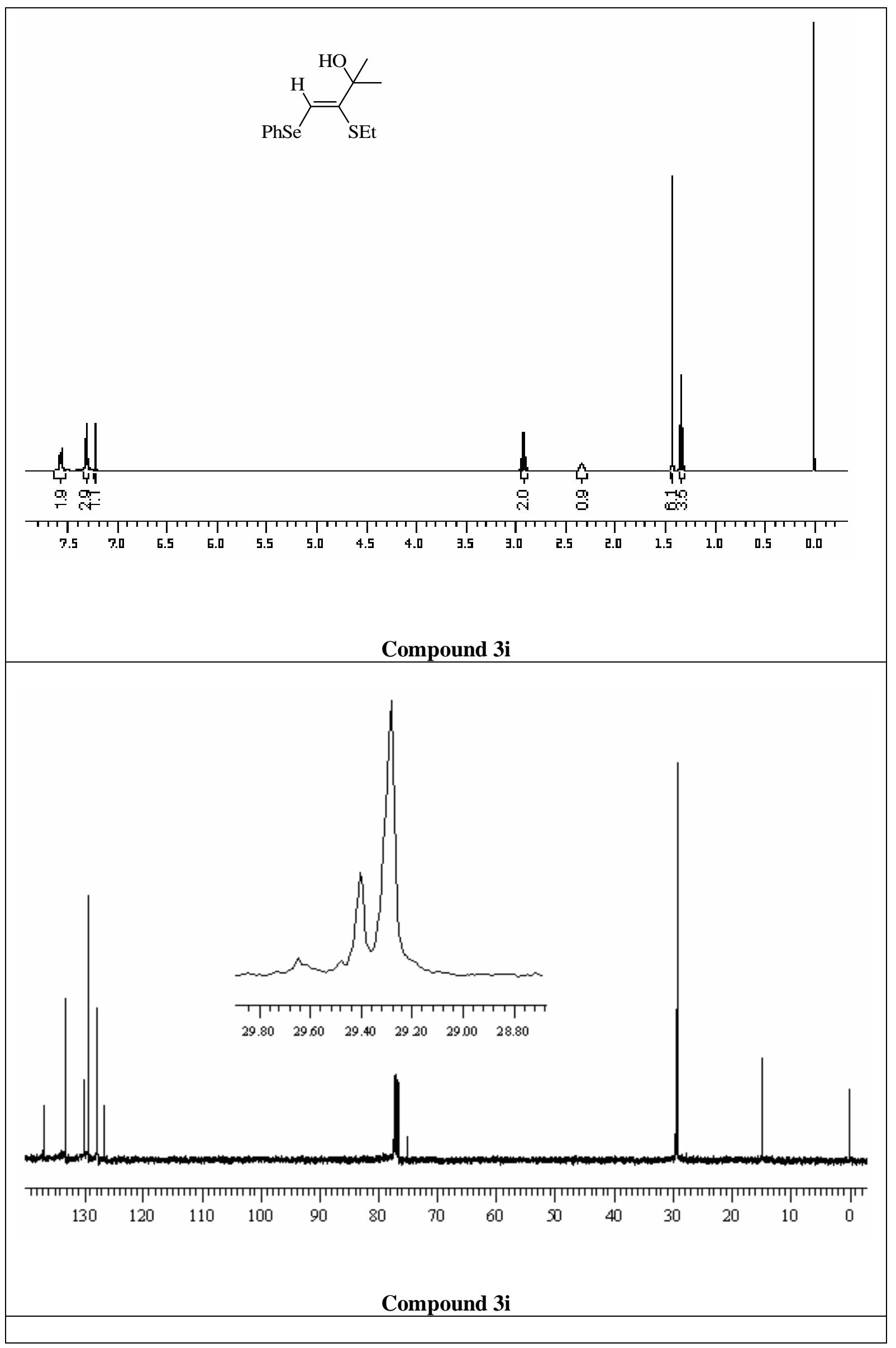




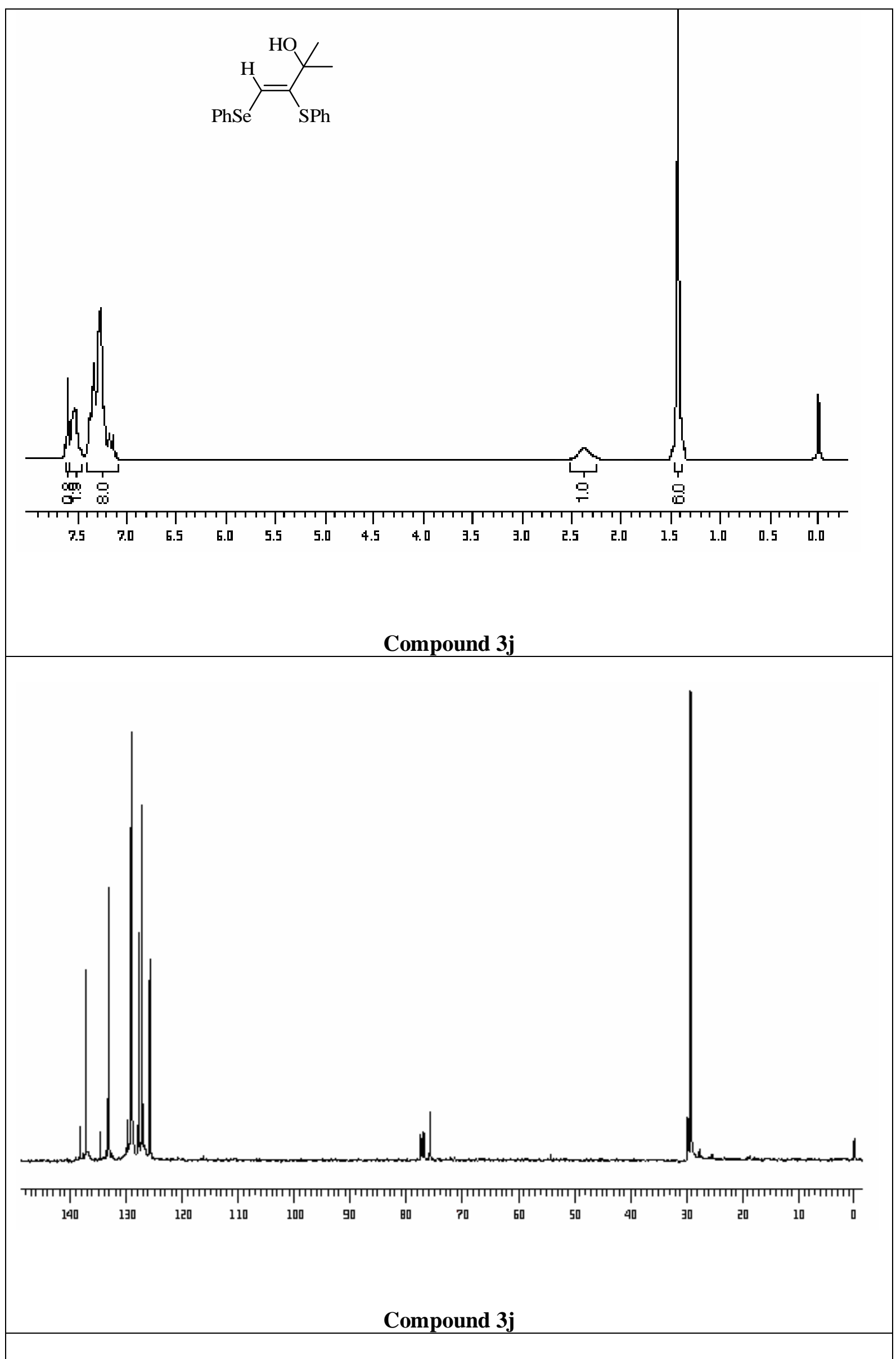




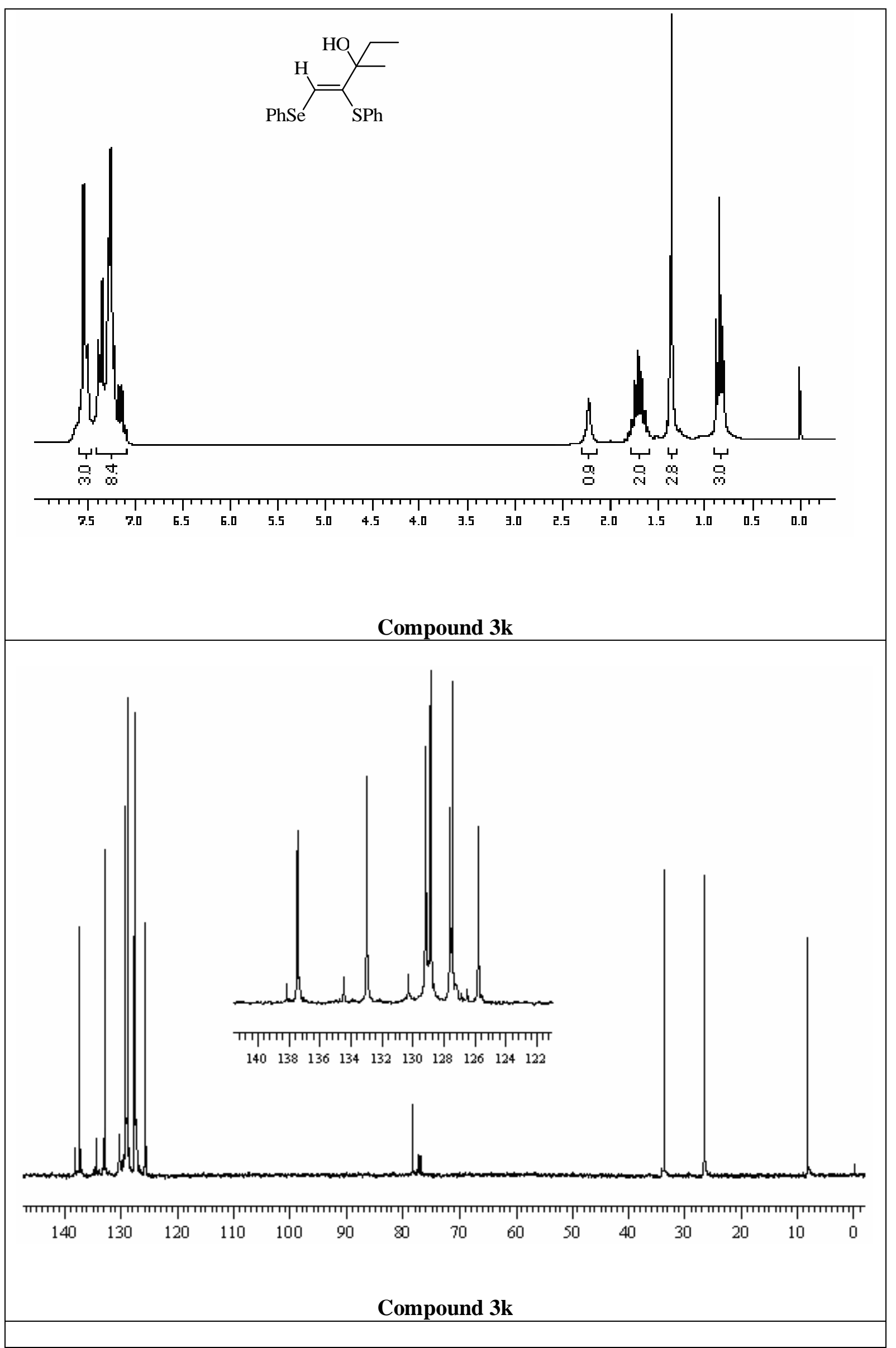




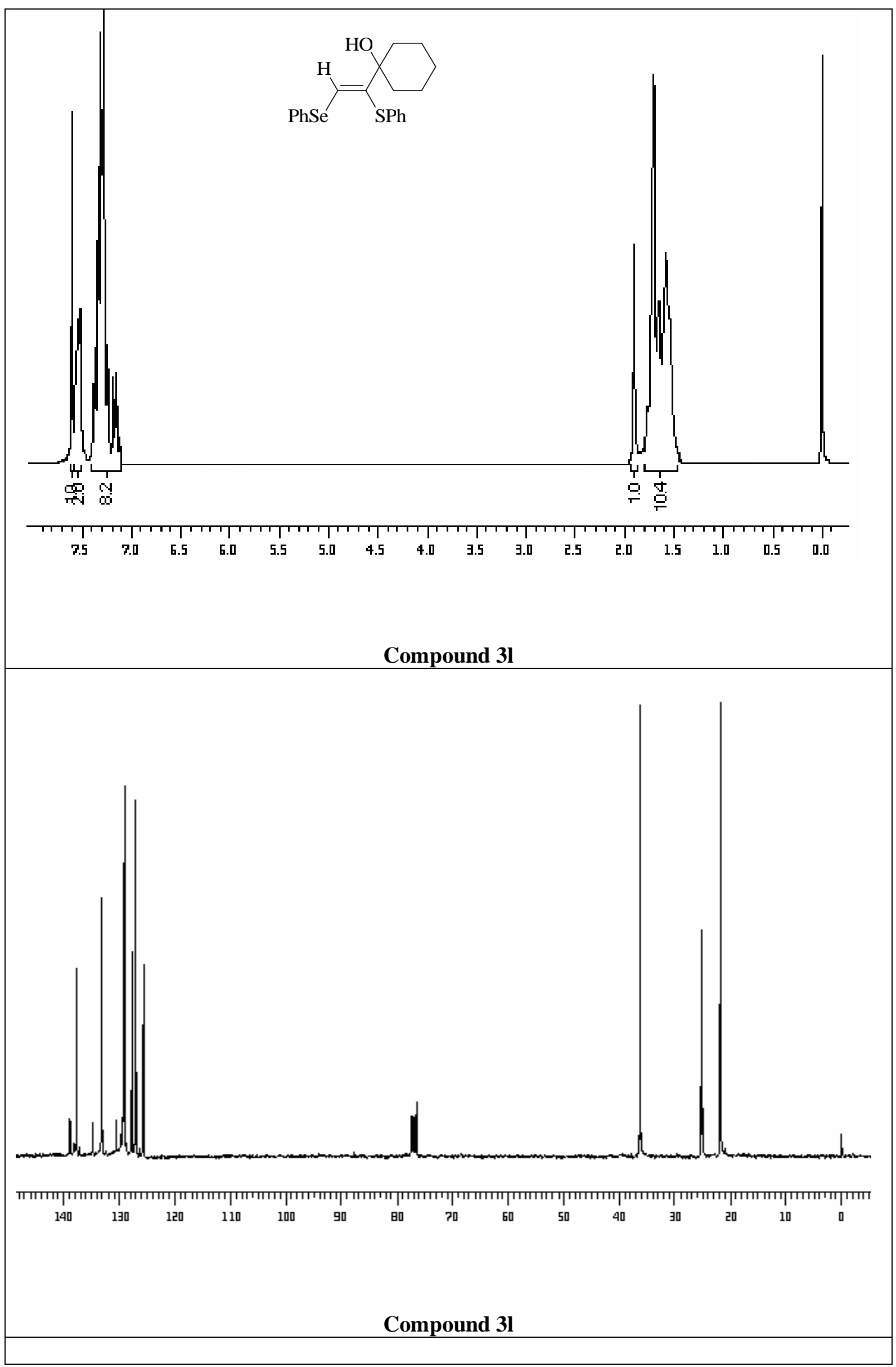




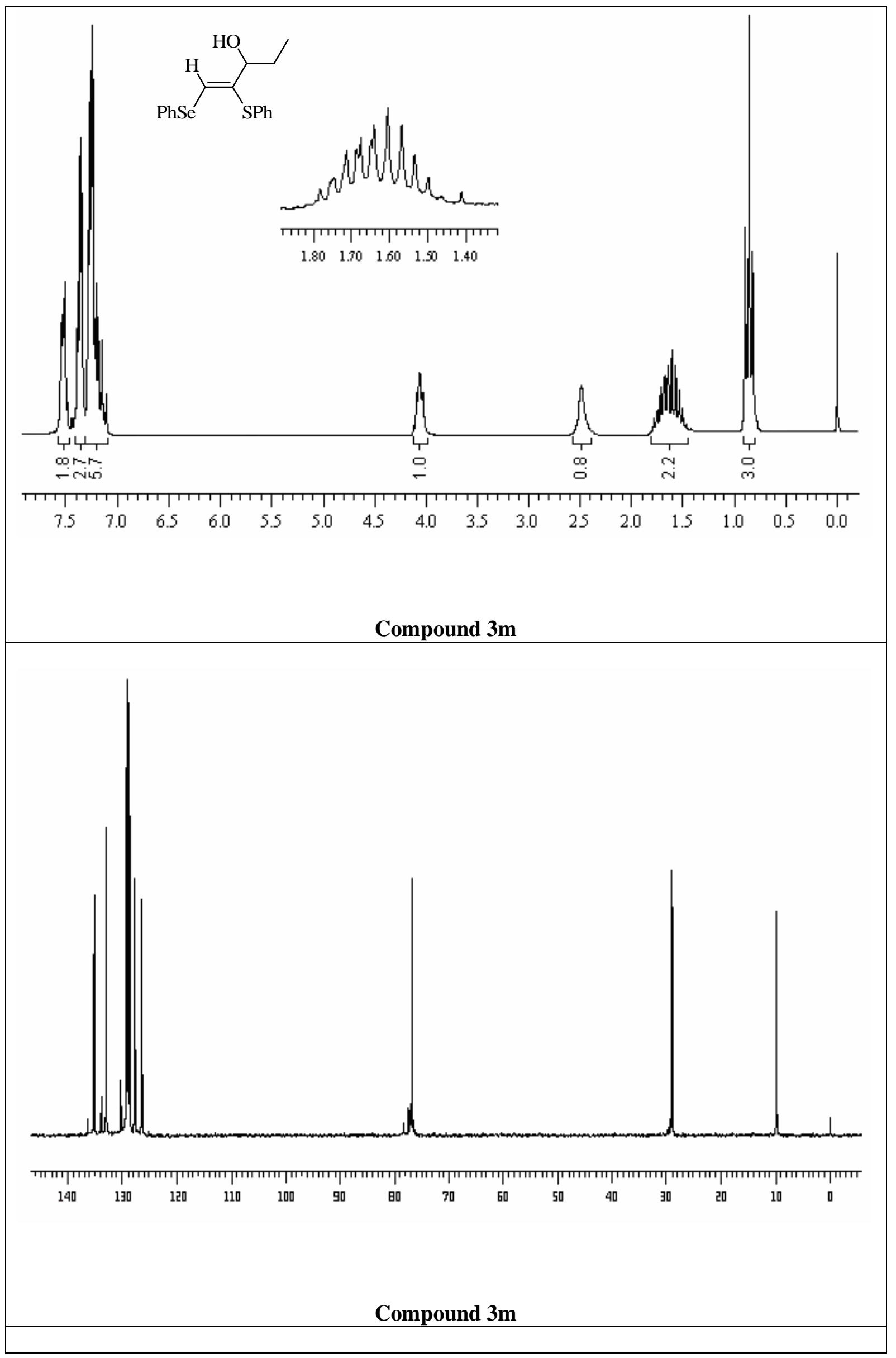




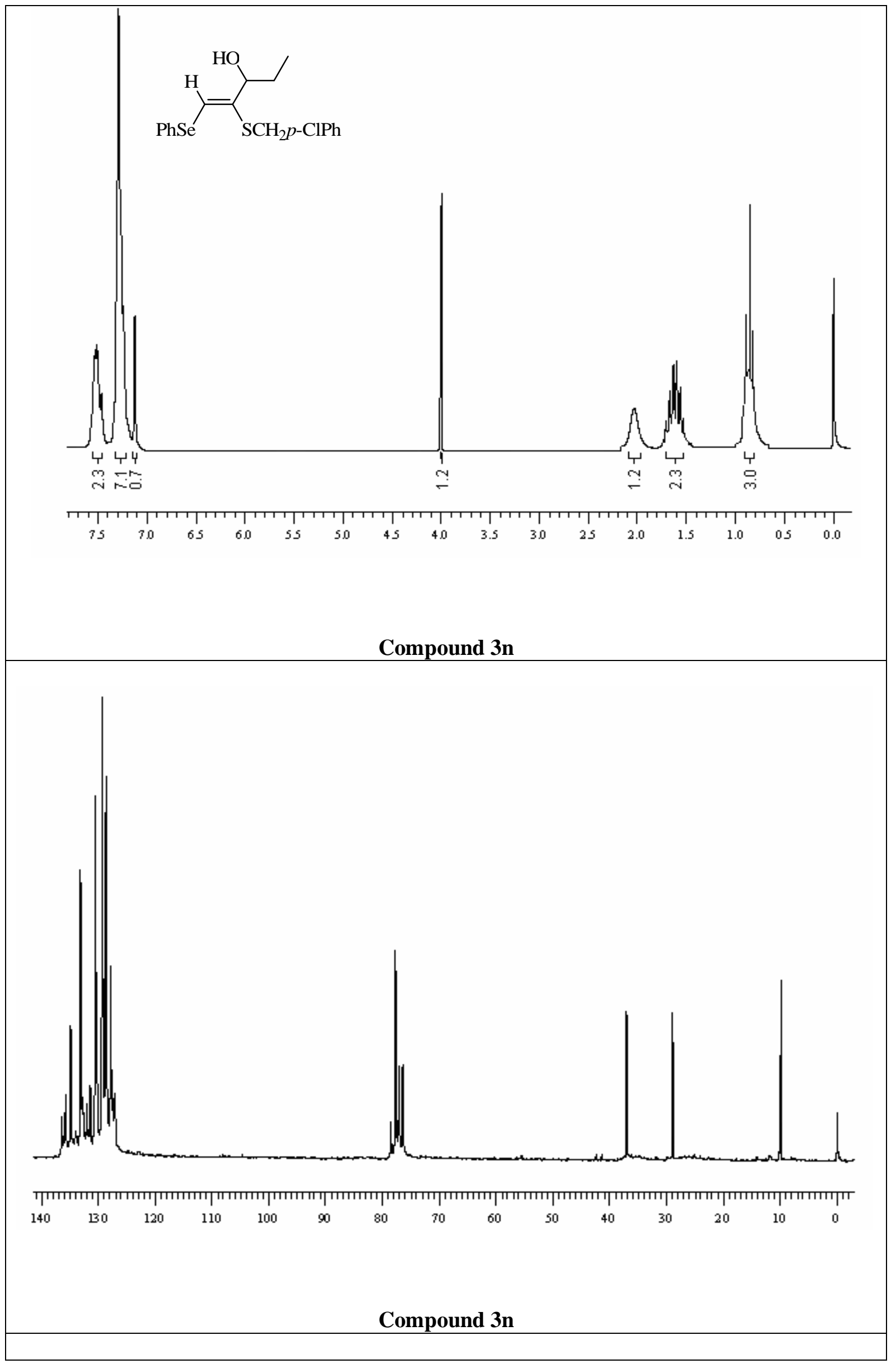




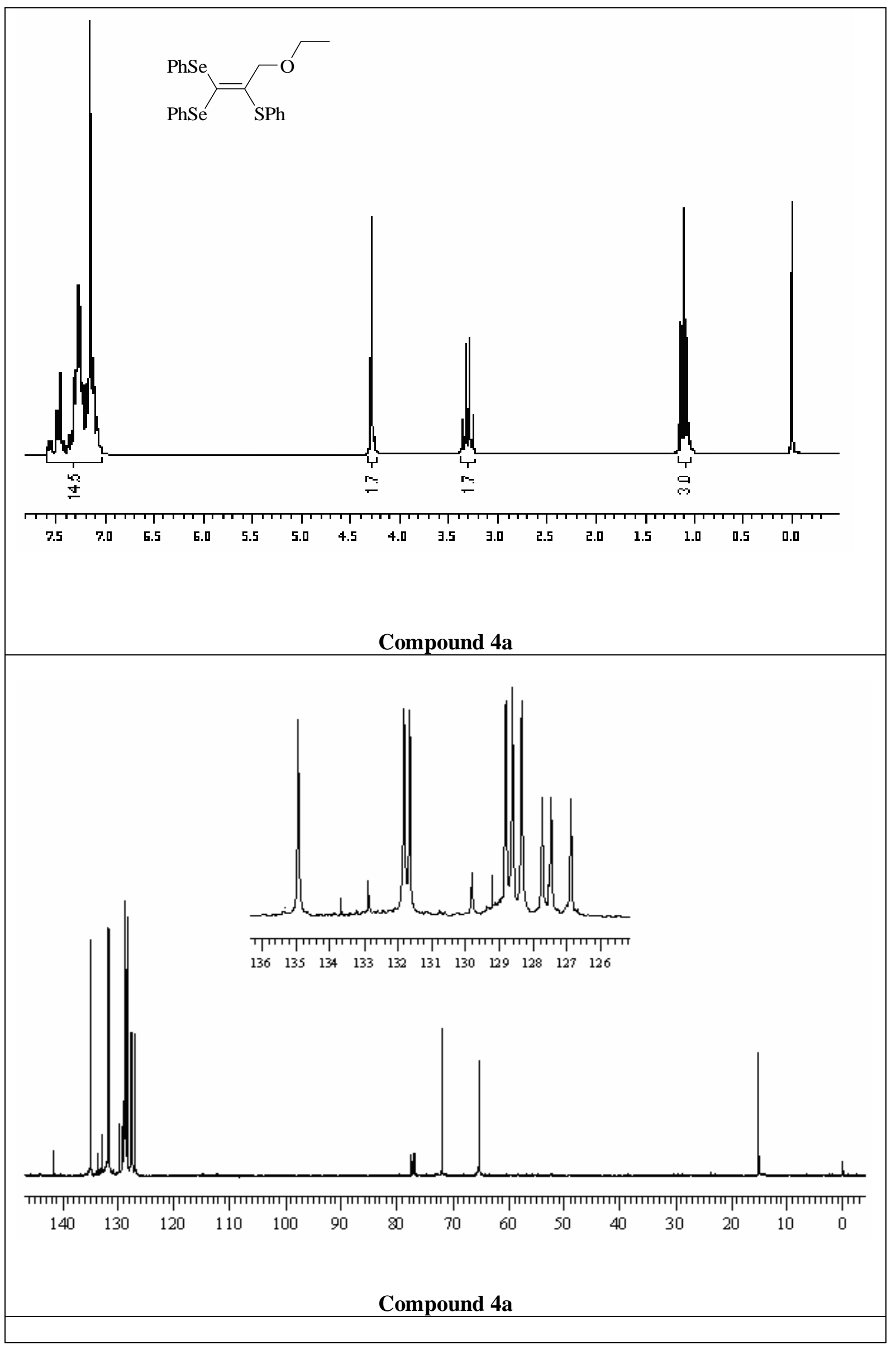




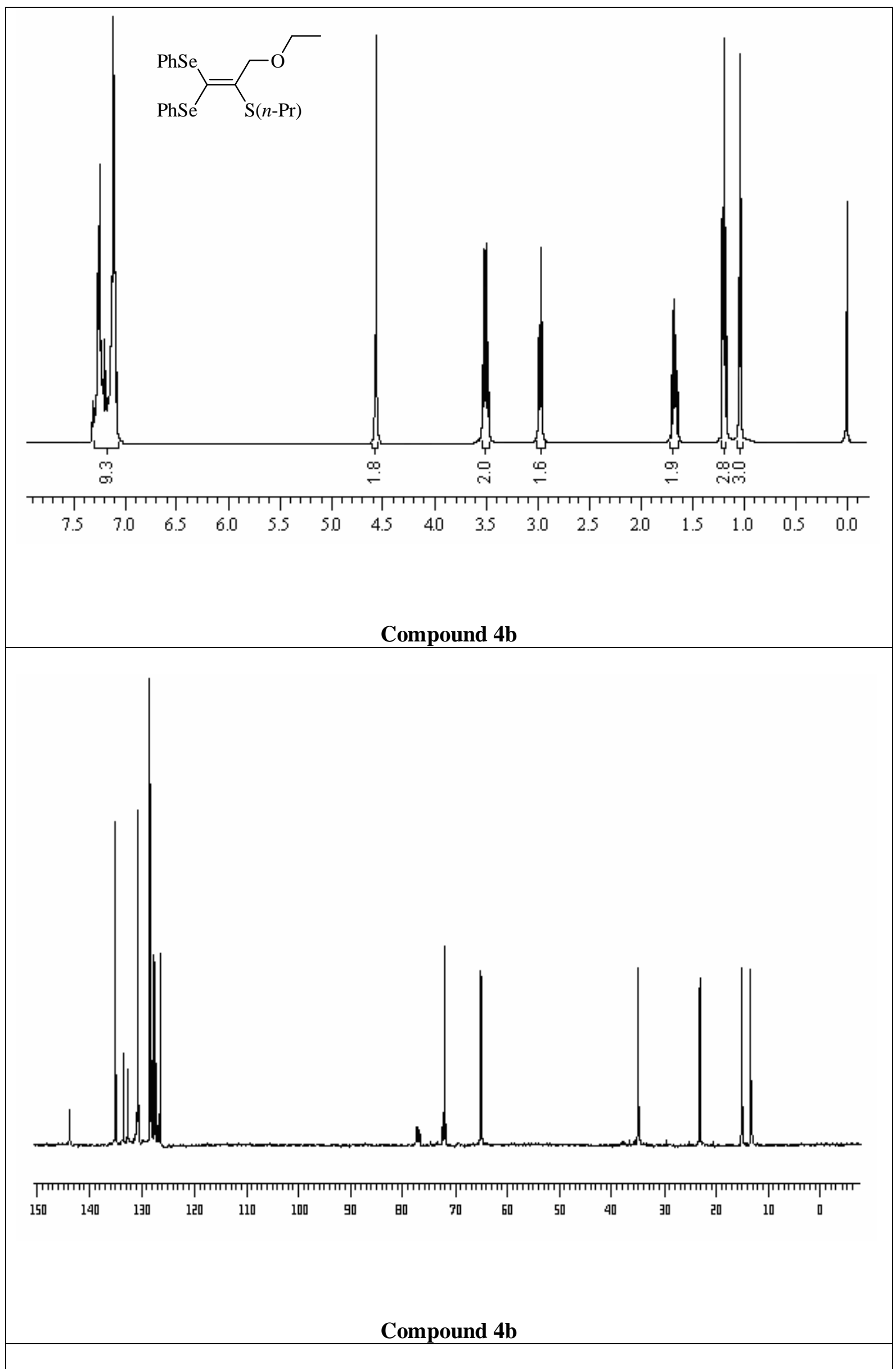




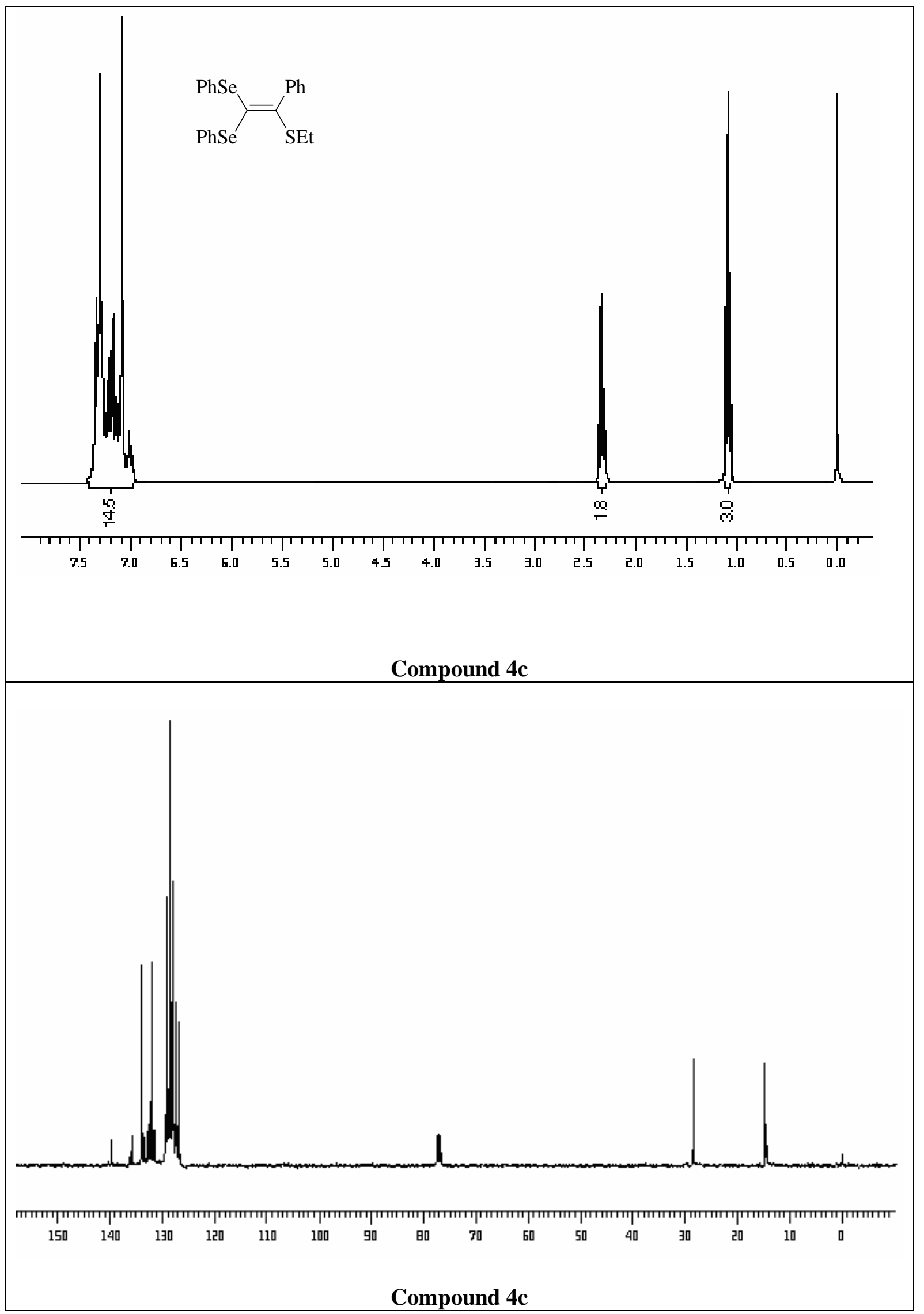


PDF created with pdfFactory trial version www.pdffactory.com 